\title{
A note on gauge anomaly cancellation in effective field theories
}

\author{
Ferruccio Feruglio \\ Dipartimento di Fisica e Astronomia 'G. Galilei', Università di Padova, \\ via Marzolo 8, I-35131 Padua, Italy \\ INFN, Sezione di Padova, \\ via Marzolo 8, I-35131 Padua, Italy \\ E-mail: feruglio@pd.infn.it
}

ABstract: The conditions for the absence of gauge anomalies in effective field theories (EFT) are rivisited. General results from the cohomology of the BRST operator do not prevent potential anomalies arising from the non-renormalizable sector, when the gauge group is not semi-simple, like in the Standard Model EFT (SMEFT). By considering a simple explicit model that mimics the SMEFT properties, we compute the anomaly in the regularized theory, including a complete set of dimension six operators. We show that the dependence of the anomaly on the non-renormalizable part can be removed by adding a local counterterm to the theory. As a result the condition for gauge anomaly cancellation is completely controlled by the charge assignment of the fermion sector, as in the renormalizable theory.

Keywords: Anomalies in Field and String Theories, Beyond Standard Model, Effective Field Theories, Gauge Symmetry

ArXiv EPrint: 2012.13989 


\section{Contents}

1 Introduction 1

2 A heuristic argument $\quad 3$

3 A miniature SMEFT $\quad 6$

$\begin{array}{lll}3.1 & \text { Four-fermion operators } & 11\end{array}$

4 Discussion $\quad 12$

\section{Introduction}

In the last few years, the role of EFT in the description of particle interactions has become even more relevant than it has been in the past, when specific scenarios like low-energy supersymmetry or composite dynamics in the electroweak symmetry breaking sector were dominating the searches for New Physics (NP). Within few assumptions concerning symmetries, field content and power counting, EFT allow to parametrize possible NP effects in the most general way. Any theory of this type includes potentially an infinite number of parameters $c_{k}$, related to the independent operators of increasing dimensionality consistent with the assumed symmetries and field content. Although information on these parameters from the experimental side is clearly crucial, it is also very important to understand whether theoretical bounds apply to them. Among such bounds, of primary importance are those related to fundamental properties of any respectable quantum field theory, such as causality, unitarity and, in the particle physics context, Lorentz invariance. Indeed, recently there has been a great activity in deriving limits on $c_{k}$ related to analyticity, unitarity and crossing properties of scattering amplitudes [1-12]. These bounds typically apply to coefficients of operators of dimension greater or equal to eight and define a permitted region, or landscape, in theory space.

Another fundamental requirement of the present description of particle interactions is gauge invariance. Strong and electroweak interactions are described by theories invariant under local continuous transformations. Gauge invariance can be realized in the exact or in the spontaneously broken phase, but in either cases it should be free from anomalies. Anomalies arise from fermion loops $[13,14]$. In renormalizable theories there is a well-known criterium for the absence of gauge anomalies [15]. It entirely relies on the transformation properties (charges and generators) of fermion fields, and it is independent from the parameters characterizing the theory. When moving from the renormalizable case to the non-rinormalizable one, comprising the whole set of EFTs, we might ask whether this criterium is still sufficient to ensure the absence of gauge anomalies, or whether it should be complemented by an additional set of conditions on the coefficients $c_{k}$. If the 
latter possibility applied, we would have discovered new bounds, sharping the landscape of admissible theories. In particular all this discussion applies to the SMEFT, which currently provides one of the most reliable tools to parametrize NP.

Given the relevance of such a question, it is not surprising that it has been discussed at length in the literature, also in relation with the SMEFT. Anomalies are non-trivial BRST cohomology classes of ghost number one, in the space of local functionals of the theory. The BRST cohomology for Yang-Mills and gravity theories coupled to scalars and fermions is known for all dimensions of space-time and for all possible local polynomial interactions, thus including the full set of possible EFT. The result is that for gravity and for semi-simple non-abelian gauge theories, all possible Lorentz-invariant anomalies are polynomials of dimension equal to the dimension of space-time [16, 17]. There are only a finite number of them for a given theory, and they are all well known. As we will argue, this strongly disfavor the possibility that gauge anomalies, for semi-simple groups, depend on the coefficients $c_{k}$ in questions. A very different result holds when there is an abelian factor in the gauge group. Then there are potential anomalies of all dimensions [18] and the dependence on $c_{k}$ requires further investigation, beyond cohomological arguments [19]. This in particular applies to the SMEFT, whose gauge group is not semi-simple.

The authors of ref. [20] computed the axial anomaly in a non-renormalizable version of quantum electrodynamics (QED), finding the same result as in renormalizable QED. Chiral anomalies in theories involving higher-derivative couplings with non-abelian gauge fields have been studied in ref. [21]. The corresponding expressions for the covariant and consistent anomalies agree with those based on the minimal Lagrangian. An extensive discussion of anomalies arising in non-renormalizable theories can be found in ref. [22]. The authors show that in $2 d$-dimensional spacetime the inclusion of a general set of local, gauge invariant, non-renormalizable operators does not lead to any new gauge anomaly, besides the ones already identified from the renormalizable part. They conclude that there is no restriction on the parameters of these operators from the requirement of gauge anomaly cancellation. Two arguments supporting this result are presented in ref. [22]. One comes from the analysis of the imaginary part of the Euclidean effective action [23]. The other one relies on the discussion of triangle diagrams and exploits two different regularization procedures. Diagrams involving only renormalizable couplings are dealt with a regulator that breaks gauge invariance, while diagrams including some non-renormalizable interaction are discussed within a gauge-invariant regularizing framework. It would be interesting to see what is the outcome when a unique regularization is used for all diagrams. This is actually one of the points illustrated in the present note. In ref. [24] anomalies arising from truncating the SMEFT at dimension six are discussed. No genuine contribution to the anomaly arises from dimension six operators. Anomaly cancellation at this order does not relate coupling constants of dimension six operators as one might naively expect, and hence does not constrain the physics beyond the SM. Nevertheless, the argument of ref. [24] cannot be considered conclusive, since the operators analyzed in this work, carrying no dependence on the Higgs field, do not form a complete set.

Dimension six operators depending on scalar fields, such as $c_{k}\left(\varphi^{\dagger} \cdot D_{\mu} \varphi-D_{\mu} \varphi^{\dagger}\right.$. $\varphi) \bar{\psi}_{k} \gamma^{\mu} \psi_{k}$, have been recently discussed in ref. [25]. The authors observed that, in the 
spontaneously broken phase, the presence of these operators leads to a shift of the fermion gauge couplings proportional to the parameters $c_{k}$. On this basis they argue that the cancellation of gauge anomalies requires new conditions involving $c_{k}$. A very recent paper [26] shows that these conditions are violated in a number of consistent, anomaly-free, models of NP. The authors of ref. [26] also show that the premature conclusion of ref. [25] derives from the non-inclusion of the (would-be) Goldstone bosons in the expression of the gauge current. This analysis, which goes in the right direction, is however still incomplete, since it does not include a general background in the computation of the anomaly. Indeed, as we shall see in detail here, the full bosonic background can reintroduce the potential dependence of the anomaly on the parameters $c_{k}$. This is an important point, perhaps the main one we would like to clarify here.

Purpose of this work is to evaluate the gauge anomaly in an EFT including a full set of dimension six-operators and allowing for the most general bosonic background, a task not yet carried out in the literature. This will be done in a simple model with U(1) gauge invariance displaying all the features of the SMEFT: i) gauge invariance realized in the broken or unbroken phase, depending on the region in parameter space; ii) a set of chiral fermions including "quarks" and "leptons"; iii) cancellation of gauge anomalies in the renormalizable part requiring a specific assignment of $U(1)$ fermion charges. We discuss the properties of the regularized effective action, obtained by integrating over the fermionic degrees of freedom. By an explicit one-loop computation, carried out within dimensional regularization, we derive the variation of this functional under a gauge transformation. The result depends on the full set of bosonic fields, scalars and gauge boson. As we shall see, even using the correct form of the current advocated in ref. [26], there are new contributions depending on $c_{k}$ and we can still wonder whether their cancellation requires a condition on $c_{k}$. The main result of the present work is to show how all the contributions depending on the coefficients of the higher-dimensional operators are trivial, since they can be canceled by adding to the regularized effective action a local polynomial in the bosonic fields. The cancellation of the remainder, non-trivial, part requires the well-known condition between the fermion charges. No other relations between Lagrangian parameters are needed.

\section{A heuristic argument}

Given a relativistic quantum field theory, renormalizable or not, invariant under the action of a gauge group $G$ and depending on a set of fields $\left(A_{\mu}, \varphi, \psi\right)$ describing particles of spin $(1,0,1 / 2)$, the gauge invariance of the related classical action $S$ can be expressed through a set of local operators $L(x):^{1}$

$$
\delta_{\alpha} S=\int d^{4} x \alpha(x) L(x) S=0 .
$$

The classical, covariantly conserved, gauge currents are defined as:

$$
j^{\mu}(x)=-\frac{1}{g} \frac{\delta S}{\delta A_{\mu}(x)} .
$$

\footnotetext{
${ }^{1}$ Indices are omitted, when not essential.
} 
In the quantized theory, anomalous contributions are caused by fermion loops and can be studied by means of the effective action $W\left[A_{\mu}, \varphi, \varphi^{\dagger}\right]$ defined through the path integral:

$$
e^{i W\left[A_{\mu}, \varphi, \varphi^{\dagger}\right]}=\int \mathcal{D} \psi \mathcal{D} \bar{\psi} e^{i S}
$$

where the integrated fields are the fermionic ones. If we are interested in the divergence of the gauge current in a general background $B=\left(A_{\mu}, \varphi, \varphi^{\dagger}\right)$ at the lowest non-trivial order, we do not need to integrate over the bosonic fields. In particular we do not address here possible contributions to the anomaly from higher loops $[27,28]$ and we do not need to quantize the fields $\left(A_{\mu}, \varphi, \varphi^{\dagger}\right)$. We will comment on this point later on. Gauge anomalies express the non-invariance of the effective action $W\left[A_{\mu}, \varphi, \varphi^{\dagger}\right]$ under gauge transformations:

$$
\delta_{\alpha} W=\int d^{4} x \alpha(x) L(x) W\left[A_{\mu}, \varphi, \varphi^{\dagger}\right] \neq 0 .
$$

Care should be taken when computing the gauge variation $L(x) W\left[A_{\mu}, \varphi, \varphi^{\dagger}\right]$. This expression is formally divergent and requires a regularization. First, what we really compute is rather:

$$
L(x) W_{\mathbf{r}}\left[A_{\mu}, \varphi, \varphi^{\dagger}\right],
$$

where $W_{\mathrm{r}}$ is the regularized version of $W .^{2}$ The renormalized effective action $W\left[A_{\mu}, \varphi, \varphi^{\dagger}\right]$ and its gauge variation are recovered by adding to $W_{\mathrm{r}}$ the space-time integral of local polynomials in the fields $\left(A_{\mu}, \varphi, \varphi^{\dagger}\right)$, thus fixing the renormalization scheme. If we can find a local polynomial $P_{\mathrm{c}}$ such that $L(x)\left(W_{\mathrm{r}}+\int d^{4} y P_{\mathrm{c}}(y)\right)=0$, we can define $W=$ $W_{\mathrm{r}}+\int d^{4} y P_{\mathrm{c}}(y)$ and the theory is free from gauge anomalies. In this case, $L(x) W_{\mathrm{r}}$ is also said an irrelevant anomaly. Thus relevant anomalies are non-trivial classes $\left\{L(x) W_{\mathrm{r}}\right\}$ under the equivalence $L(x) W_{\mathrm{r}} \sim L(x) W_{\mathrm{r}}^{\prime}$ where $W_{\mathrm{r}}^{\prime}=W_{\mathrm{r}}+\int d^{4} y P(y), P(y)$ being a local polynomial in the bosonic fields. In general, we expect $L(x) W_{\mathrm{r}}$ to contain relevant and irrelevant contributions.

The functional dependence of $L(x) W_{\mathrm{r}}$, is strongly constrained by the Wess-Zumino consistency conditions [29]:

$$
L_{a}(x) L_{b}(y) W_{\mathrm{r}}-L_{b}(y) L_{a}(x) W_{\mathrm{r}}=\delta^{4}(x-y) f_{a b}^{c} L_{c}(x) W_{\mathrm{r}}
$$

consequence of the algebra of the operators $L(x)$. Here $f_{a b}^{c}$ are the structure constants of the gauge group. We can regard the class $\left\{L_{a}(x) W_{\mathrm{r}}\right\}$ as the unknown in eq. (2.6). The general solution can be derived from cohomological arguments. ${ }^{3}$ For semi-simple nonabelian gauge theories, renormalizable or not, all possible Lorentz-invariant anomalies are polynomials of dimension equal to the dimension of space-time [17]. They coincide with the well-known Adler-Bell-Jackiw anomalies [16]. Moreover, by the non-renormalization theorem of Adler-Bardeen [27] and its generalization to non-renormalizable theories [28], such anomalies are exhausted by one-loop contributions. These results are the ingredients

\footnotetext{
${ }^{2} \mathrm{~A}$ limiting procedure where the regulator is removed after the evaluation of $L(x) W_{\mathrm{r}}\left[A_{\mu}, \varphi, \varphi^{\dagger}\right]$ is understood here.

${ }^{3}$ In the fully quantized theory, gauge invariance is replaced by BRST invariance, and eq. (2.6) is replaced by $\delta_{B R S T}^{2} W_{\mathrm{r}}=0, \delta_{B R S T}$ being the nilpotent BRST operator.
} 
of a heuristic argument excluding the dependence of the anomaly on the coefficients $c_{k}$ controlling non-renormalizable operators, for semi-simple gauge groups.

The argument goes as follows. In a general EFT the relevant fermion bilinear interaction reads:

$$
\bar{f}\left(\mathscr{S}+\mathscr{P}_{\gamma_{5}}+\mathscr{V}_{\mu} \gamma^{\mu}+\mathscr{A}_{\mu} \gamma^{\mu} \gamma_{5}+\mathscr{T}_{\mu \nu} \sigma^{\mu \nu}\right) f,
$$

where $\mathscr{S}, \mathscr{P}, \mathscr{V}_{\mu}, \mathscr{A}_{\mu}, \mathscr{T}_{\mu \nu}$ are polynomials in the bosonic fields and their derivatives. Only the $\mathscr{V}_{\mu}, \mathscr{A}_{\mu}$ couplings matter for the anomaly. We can expand them in contributions coming from operators of increasing dimensionality:

$$
\begin{array}{ll}
\mathscr{V}_{\mu}=c_{0}^{V} A_{\mu}+\Omega_{\mu}^{V} & \Omega_{\mu}^{V}=\sum_{k>0} c_{k}^{V} \mathcal{O}_{k, \mu}^{V} \\
\mathscr{A}_{\mu}=c_{0}^{A} A_{\mu}+\Omega_{\mu}^{A} & \Omega_{\mu}^{A}=\sum_{k>0} c_{k}^{A} \mathcal{O}_{k, \mu}^{A} .
\end{array}
$$

Here the coefficients $c_{0}^{V, A}$ come from the renormalizable part and have zero mass dimension. They are fixed by the minimal coupling between fermions and gauge bosons, completely determined by the gauge transformation properties of the fermion fields. The parameters $c_{k}^{V, A}(k>0)$ derive from the non-renormalizable sector and have mass dimension $-k$. The operators $\mathcal{O}_{k, \mu}^{V, A}$ have dimension $k+1$. The functional $W_{\mathrm{r}}$, evaluated at one-loop order, can be expanded in powers of $\mathscr{V}_{\mu}$ and $\mathscr{A}_{\mu}$. The gauge variation of any given order of such an expansion can be decomposed into two parts. The first one depends only on the gauge field $A_{\mu}$. If not vanishing, this part should be a polynomial of degree four in $A_{\mu}$ and its derivatives. The second one originates also from $\Omega_{\mu}^{V, A}$ and will contain some monomial in fields and derivatives of degree higher than four, contrary to the general cohomological results. Therefore either these new monomials are vanishing or they are irrelevant, and we conclude that there is no dependence on $c_{k}^{V, A}(k>0)$ in the anomaly.

In this work we will not rely on the previous heuristic argument, since in any case it does not apply to non semi-simple gauge groups like the one of the SMEFT. The solutions of the Wess-Zumino consistency conditions in the abelian case allow potential anomalies of any dimensionality. Indeed, the right-hand side of eq. (2.6) vanishes for abelian groups and $L(x) W_{\mathrm{r}}$ can be any gauge invariant polynomial in $A_{\mu}, \varphi, \varphi^{\dagger}$, whose dimension is not bounded. It is not difficult to build some candidates for relevant anomalies $\left\{L(x) W_{\mathrm{r}}\right\}$. Consider a $\mathrm{U}(1)$ gauge theory where $\varphi$ is a complex scalar field carrying a non-vanishing charge. The following local operators:

$$
\varphi^{\dagger} \varphi \varepsilon^{\mu \nu \rho \sigma} \partial_{\mu} A_{\nu} \partial_{\rho} A_{\sigma}, \quad i \varphi^{\dagger} \varphi \varepsilon^{\mu \nu \rho \sigma} \partial_{\mu}\left(\varphi^{\dagger} D_{\nu} \varphi-D_{\nu} \varphi^{\dagger} \varphi\right) \partial_{\rho} A_{\sigma},
$$

are solutions of eq. (2.6) and cannot be expressed as gauge variations of an integrated local polynomial. The previous heuristic argument does not apply to these examples. These expressions can only come from the contribution of higher-dimensional operators to the anomaly and, if present, carry a dependence on the coefficients $c_{k}$. Their occurrence in a given model can only be verified through a direct computation, which constitutes the main aim of this paper. By considering a simple explicit model that mimics the SMEFT properties, we will see that $L(x) W_{\mathrm{r}}$ is the sum of two contributions. The first one includes 
only the gauge fields $A_{\mu}$ and is independent from the Lagrangian coefficients $c_{k}$. It leads to the well-known conditions for gauge anomaly cancellation. The second one involves both $A_{\mu}$ and $\varphi$ and depends on the coefficients $c_{k}$. We explicitly show that this part is irrelevant and does not require additional conditions for the absence of gauge anomalies. A posteriori, this provides a confirmation of the above heuristic argument, at least in a specific case.

\section{A miniature SMEFT}

To illustrate the mechanism of anomaly cancellations in EFT, we consider a toy model displaying many features of the SMEFT, but sufficiently simple to allow a concise description of the problem. The model enjoys a $\mathrm{U}(1)_{Q}$ gauge symmetry. The matter fields consist of two four-component fermions, $l$ and $q$, mimicking leptons and quarks in the SMEFT, plus a complex scalar $\varphi$, the analogue of the SMEFT Higgs multiplet. In a four-component notation, $Q\left(l_{L}\right)=-Q\left(q_{L}\right)=Q(\varphi)=-1$ and $Q\left(l_{R}\right)=Q\left(q_{R}\right)=0$. The gauge theory is chiral but with this assignment gauge anomalies generated by the renormalizable part of the theory are absent. In what follows we will focus on the sector of the theory where baryon and lepton numbers $B$ and $L$ are conserved. ${ }^{4}$ The effective Lagrangian reads:

$$
\mathcal{L}=\mathcal{L}_{4}+\mathcal{L}_{6}+\ldots
$$

where $\mathcal{L}_{4}$ denotes the renormalizable part, $\mathcal{L}_{6}$ collects dimension six operators and dots stand for higher-dimensional contributions. We have:

$$
\begin{aligned}
\mathcal{L}_{4}= & -\frac{1}{4} F_{\mu \nu} F^{\mu \nu}+\overline{l_{L}} i \gamma^{\mu} D_{\mu} l_{L}+\overline{l_{R}} i \gamma^{\mu} \partial_{\mu} l_{R}+\overline{q_{L}} i \gamma^{\mu} D_{\mu} q_{L}+\overline{q_{R}} i \gamma^{\mu} \partial_{\mu} q_{R} \\
& +D_{\mu} \varphi^{\dagger} D^{\mu} \varphi-V\left(\varphi^{\dagger} \varphi\right)-\left(y_{l} \varphi \overline{l_{L}} l_{R}+y_{q} \varphi^{\dagger} \overline{q_{L}} q_{R}+\text { h.c. }\right)
\end{aligned}
$$

where $D_{\mu} \psi=\left(\partial_{\mu}+i g Q(\psi) A_{\mu}\right) \psi,\left(\psi=l_{L}, l_{R}, q_{L}, q_{R}, \varphi\right)$ and $V\left(\varphi^{\dagger} \varphi\right)=\mu^{2}\left(\varphi^{\dagger} \varphi\right)+\lambda\left(\varphi^{\dagger} \varphi\right)^{2}$ $(\lambda>0)$. Depending on the sign of $\mu^{2}$, the gauge symmetry is spontaneously broken or not. When $\mu^{2}<0\left(\mu^{2}>0\right)$ the theory is in the broken(unbroken) phase. We will discuss both cases at once. At dimension six we have [30]:

$$
\mathcal{L}_{6}=\sum_{k} c_{k} O_{k}+\ldots
$$

where $O_{k}$ are the operators in table 1 , dots stand for four-fermion operators, that will be discussed in section 3.1. The coefficients $c_{k}$ have mass dimension -2 and implicitly carry the dependence on some reference scale $\Lambda .{ }^{5}$ We define the classical current $j^{\mu}$ as:

$$
j^{\mu}(x)=-\frac{1}{g} \frac{\delta S}{\delta A_{\mu}(x)} .
$$

\footnotetext{
${ }^{4}$ At variance with the SM, the conservation of $B$ and $L$ at the renormalizable level are not automatic in our setting, but it could be easily enforced by suitable discrete symmetries.

${ }^{5}$ It is customary to set: $c_{k}=\tilde{c}_{k} / \Lambda^{2}$, where $\tilde{c}_{k}$ are dimensionless.
} 


\begin{tabular}{|c|c|}
\hline$O_{\varphi}$ & $\left(\varphi^{\dagger} \varphi\right)^{3}$ \\
\hline$O_{\varphi \square}$ & $\left(\varphi^{\dagger} \varphi\right) \square\left(\varphi^{\dagger} \varphi\right)$ \\
\hline$O_{\varphi D}$ & $\left(\varphi^{\dagger} D^{\mu} \varphi\right)^{*}\left(\varphi^{\dagger} D_{\mu} \varphi\right)$ \\
\hline$O_{\varphi A}$ & $g^{2}\left(\varphi^{\dagger} \varphi\right) F_{\mu \nu} F^{\mu \nu}$ \\
\hline$O_{\varphi \widetilde{A}}$ & $g^{2}\left(\varphi^{\dagger} \varphi\right) F_{\mu \nu} \widetilde{F}^{\mu \nu}$ \\
\hline
\end{tabular}

\begin{tabular}{|c|c|}
\hline$O_{l \varphi}$ & $\left(\varphi^{\dagger} \varphi\right) \varphi \overline{l_{L}} l_{R}$ \\
\hline$O_{q \varphi}$ & $\left(\varphi^{\dagger} \varphi\right) \varphi^{\dagger} \overline{q_{L}} q_{R}$ \\
\hline$O_{l A}$ & $g \varphi \overline{l_{L}} \sigma^{\mu \nu} l_{R} F_{\mu \nu}$ \\
\hline$O_{q A}$ & $g \varphi^{\dagger} \overline{q_{L}} \sigma^{\mu \nu} q_{R} F_{\mu \nu}$ \\
\hline$O_{\varphi l_{L}}$ & $i\left(\varphi^{\dagger} \stackrel{\leftrightarrow}{D}_{\mu} \varphi\right) \overline{l_{L}} \gamma^{\mu} l_{L}$ \\
\hline$O_{\varphi l_{R}}$ & $i\left(\varphi^{\dagger} \stackrel{\leftrightarrow}{D}_{\mu} \varphi\right) \overline{l_{R}} \gamma^{\mu} l_{R}$ \\
\hline$O_{\varphi q_{L}}$ & $i\left(\varphi^{\dagger} \stackrel{\leftrightarrow}{D}_{\mu} \varphi\right) \overline{q_{L}} \gamma^{\mu} q_{L}$ \\
\hline$O_{\varphi q_{R}}$ & $i\left(\varphi^{\dagger} \stackrel{\leftrightarrow}{D}_{\mu} \varphi\right) \overline{q_{R}} \gamma^{\mu} q_{R}$ \\
\hline
\end{tabular}

Table 1. Dimension-six operators other than the four-fermion ones.

Here $S$ is the classical action and the derivative is the variational one. We have:

$$
\begin{aligned}
j^{\mu}= & j_{3}^{\mu}+j_{5}^{\mu}, \\
j_{3}^{\mu}= & \partial_{\lambda} F^{\lambda \mu}-\overline{l_{L}} \gamma^{\mu} l_{L}+\overline{q_{L}} \gamma^{\mu} q_{L}-i\left(\varphi^{\dagger} \stackrel{\leftrightarrow}{D} \varphi\right), \\
j_{5}^{\mu}= & -i c_{\varphi D} \varphi^{\dagger} \varphi\left(\varphi^{\dagger} \stackrel{\leftrightarrow}{D} \varphi\right)+4 g c_{\varphi A} \partial_{\lambda}\left(\varphi^{\dagger} \varphi F^{\lambda \mu}\right)+4 g c_{\varphi} \widetilde{A}_{\lambda}\left(\varphi^{\dagger} \varphi \widetilde{F}^{\lambda \mu}\right) \\
& +2 c_{l A} \partial_{\lambda}\left(\varphi \overline{l_{L}} \sigma^{\lambda \mu} l_{R}\right)+2 c_{q A} \partial_{\lambda}\left(\varphi^{\dagger} \overline{q_{L}} \sigma^{\lambda \mu} q_{R}\right)-2 \varphi^{\dagger} \varphi \sum_{i} c_{\varphi f_{i}} \bar{f}_{i} \gamma^{\mu} f_{i} .
\end{aligned}
$$

From the invariance of the classical action under infinitesimal gauge transformations $\delta_{\alpha} A_{\mu}(x)$ and $\delta_{\alpha} \chi_{I}(x), \chi_{I}(x)$ denoting collectively the matter fields, we get:

$$
\delta_{\alpha} S=\int d^{4} x\left[\frac{\delta S}{\delta A_{\mu}(x)} \delta_{\alpha} A_{\mu}(x)+\frac{\delta S}{\delta \chi_{I}(x)} \delta_{\alpha} \chi_{I}(x)\right]=0,
$$

Along the solutions of the equations of motion, where the second term vanishes, we recover the conservation of the current $j_{\mu}(x)(3.5),(3.6)$ in the classical theory.

We analyze the anomalous contributions caused by fermion loops by means of the effective action $W\left[A_{\mu}, \varphi, \varphi^{\dagger}\right]$ defined in eq. (2.3). The infinitesimal variation of a generic functional $F\left[A_{\mu}, \varphi, \varphi^{\dagger}\right]$ under gauge transformations of the fields $A_{\mu}, \varphi$ and $\varphi^{\dagger}$ can be expressed trough the operator $L(x)$ :

$$
\delta_{\alpha} F=\int d^{4} x \alpha(x) L(x) F \quad L(x)=\left[-\frac{1}{g} \partial_{\mu} \frac{\delta}{\delta A_{\mu}(x)}+i \varphi(x) \frac{\delta}{\delta \varphi(x)}-i \varphi^{\dagger}(x) \frac{\delta}{\delta \varphi^{\dagger}(x)}\right] .
$$

From the invariance of the action $S$ under gauge transformations, the following identity follows:

$$
L(x) W\left[A_{\mu}, \varphi, \varphi^{\dagger}\right]=\operatorname{Anomaly}(x),
$$


where the anomaly is a local polynomial in the bosonic fields $B=\left(A_{\mu}, \varphi, \varphi^{\dagger}\right)$, which vanishes provided the measure is invariant under a gauge transformation of the fermionic sector. ${ }^{6}$

This is precisely the issue analyzed here. When $\mathcal{L}_{6}$ is set to zero, the condition for anomaly cancellation, $Q\left(l_{L}\right)+Q\left(q_{L}\right)=0$, is automatically satisfied and eq. (3.9) holds with vanishing right-hand-side. We would like to check whether the cancellation of the gauge anomaly requires additional conditions on the coefficients $c_{k}$, when $\mathcal{L}_{6}$ is turned on. To this purpose we directly compute the functional $W$, controlled by the interaction involving fermion bilinear terms:

$$
\mathcal{L}_{\bar{f} f}=\bar{f}\left(\mathscr{S}+\mathscr{P} \gamma_{5}+\mathscr{V}_{\mu} \gamma^{\mu}+\mathscr{A}_{\mu} \gamma^{\mu} \gamma_{5}+\mathscr{T}_{\mu \nu} \sigma^{\mu \nu}\right) f
$$

where $f=(l, q)^{T}$ and:

$$
\begin{aligned}
\mathscr{S} & =\frac{1}{2}\left(\mathscr{Y}+\mathscr{Y}^{\dagger}\right) \quad \mathscr{P}=\frac{1}{2}\left(\mathscr{Y}-\mathscr{Y}^{\dagger}\right) \\
\mathscr{V}_{\mu} & =\frac{1}{2}\left[-g Q A_{\mu}+i\left(C_{\phi R}+C_{\phi L}\right)\left(\varphi^{\dagger} \stackrel{\leftrightarrow}{D}_{\mu} \varphi\right)\right] \\
\mathscr{A}_{\mu} & =\frac{1}{2}\left[+g Q A_{\mu}+i\left(C_{\phi R}-C_{\phi L}\right)\left(\varphi^{\dagger} \stackrel{\leftrightarrow}{D}_{\mu} \varphi\right)\right] \\
\mathscr{T}_{\mu \nu} & =\frac{g}{2}\left[\left(\Sigma+\Sigma^{\dagger}\right) F_{\mu \nu}+i\left(\Sigma-\Sigma^{\dagger}\right) \widetilde{F}_{\mu \nu}\right],
\end{aligned}
$$

The quantities $Q, C_{\phi R}, C_{\phi L}$ are field-independent matrices, whereas $\mathscr{Y}$, and $\Sigma$ are matrices depending on the scalar field $\varphi$ and its conjugate:

$$
\begin{array}{rlrl}
Q=\left(\begin{array}{cc}
-1 & 0 \\
0 & +1
\end{array}\right) & C_{\phi R(L)} & =\left(\begin{array}{cc}
c_{\varphi l_{L(R)}} & 0 \\
0 & c_{\varphi q_{L(R)}}
\end{array}\right) \\
\mathscr{Y}=\left(\begin{array}{cc}
-y_{l} \varphi+c_{l \varphi} \varphi^{\dagger} \varphi \varphi & 0 \\
0 & -y_{q} \varphi+c_{q \varphi} \varphi^{\dagger} \varphi \varphi
\end{array}\right) & \Sigma=\left(\begin{array}{cc}
c_{l A} \varphi & 0 \\
0 & c_{q A} \varphi^{\dagger}
\end{array}\right) .
\end{array}
$$

The functional $W$ depends on $A_{\mu}, \varphi, \varphi^{\dagger}$ through the combinations $\mathscr{S}, \mathscr{P}, \mathscr{V}_{\mu}, \mathscr{A}_{\mu}$ and $\mathscr{T}_{\mu \nu}$. To check the gauge invariance of $W$, we Taylor expand $W$ in powers of these combinations. Since only triangle diagrams with $\mathscr{V}_{\mu}$ and $\mathscr{A}_{\mu}$ insertions can contribute to the anomaly [3133], we only need to consider the following term:

$$
\begin{aligned}
W=\int d^{4} x_{1} d^{4} x_{2} d^{4} x_{3}\{ & \frac{1}{3 !} W_{\mu \nu \lambda}^{L L}\left(x_{1}, x_{2}, x_{3}\right) \operatorname{tr}\left[\mathscr{L}_{\mu}\left(x_{1}\right) \mathscr{L}_{\nu}\left(x_{2}\right) \mathscr{L}_{\lambda}\left(x_{3}\right)\right] \\
& +\frac{1}{2 !} W_{\mu \nu \lambda}^{L L R}\left(x_{1}, x_{2}, x_{3}\right) \operatorname{tr}\left[\mathscr{L}_{\mu}\left(x_{1}\right) \mathscr{L}_{\nu}\left(x_{2}\right) \mathscr{R}_{\lambda}\left(x_{3}\right)\right] \\
& +\frac{1}{2 !} W_{\mu \nu \lambda}^{L R R}\left(x_{1}, x_{2}, x_{3}\right) \operatorname{tr}\left[\mathscr{L}_{\mu}\left(x_{1}\right) \mathscr{R}_{\nu}\left(x_{2}\right) \mathscr{R}_{\lambda}\left(x_{3}\right)\right] \\
& \left.+\frac{1}{3 !} W_{\mu \nu \lambda}^{R R R}\left(x_{1}, x_{2}, x_{3}\right) \operatorname{tr}\left[\mathscr{R}_{\mu}\left(x_{1}\right) \mathscr{R}_{\nu}\left(x_{2}\right) \mathscr{R}_{\lambda}\left(x_{3}\right)\right]\right\}+\ldots
\end{aligned}
$$

\footnotetext{
${ }^{6}$ An equivalent, more familiar, statement is $\partial_{\mu}\left\langle j^{\mu}(x)\right\rangle_{B}+i \varphi(x) \frac{\delta W}{\delta \varphi(x)}-i \varphi(x)^{\dagger} \frac{\delta W}{\delta \varphi(x)^{\dagger}}=$ Anomaly, where, by definition, $\left\langle j^{\mu}(x)\right\rangle_{B} \equiv-\frac{1}{g} \frac{\delta W}{\delta A_{\mu}(x)}$.
} 
where we have defined:

$$
\mathscr{R}_{\mu}(x)=\mathscr{V}_{\mu}(x)+\mathscr{A}_{\mu}(x), \quad \mathscr{L}_{\mu}(x)=\mathscr{V}_{\mu}(x)-\mathscr{A}_{\mu}(x)
$$

The coefficients $W_{\mu \nu \lambda}^{X Y Z}\left(x_{1}, x_{2}, x_{3}\right)$ are field-independent functions of the space-time points $x_{1,2,3}$, that can be evaluated by a one-loop computation. The function $W_{\mu \nu \lambda}^{L L L}\left(x_{1}, x_{2}, x_{3}\right)$ is symmetric under permutations of $\left(\mu, x_{1}\right),\left(\nu, x_{2}\right)$ and $\left(\lambda, x_{3}\right)$. Analogous properties hold for the other expressions. We also define $W_{\nu \mu \lambda}^{Y X Z}\left(x_{2}, x_{1}, x_{3}\right)=W_{\mu \nu \lambda}^{X Y Z}\left(x_{1}, x_{2}, x_{3}\right)$ and so on. An important property of the operator $L(x)$ is:

$$
L(x) \mathscr{L}_{\mu}(y)=Q \partial_{\mu} \delta^{4}(x-y), \quad L(x) \mathscr{R}_{\mu}(y)=0 .
$$

This is a consequence of the gauge invariance of the combination $i\left(\varphi^{\dagger} \stackrel{\leftrightarrow}{D}_{\mu} \varphi\right)$ contributing to $\mathscr{V}_{\mu}$ and $\mathscr{A}_{\mu}$. We get:

$$
\begin{aligned}
L(x) W=\frac{1}{2} \int d^{4} y d^{4} z & \left\{\partial^{\mu} W_{\mu \nu \lambda}^{L L L}(x, y, z) \operatorname{tr}\left[Q \mathscr{L}_{\nu}(y) \mathscr{L}_{\lambda}(z)\right]\right. \\
& +2 \partial^{\mu} W_{\mu \nu \lambda}^{L L R}(x, y, z) \operatorname{tr}\left[Q \mathscr{L}_{\nu}(y) \mathscr{R}_{\lambda}(z)\right] \\
& \left.+\partial^{\mu} W_{\mu \nu \lambda}^{L R R}(x, y, z) \operatorname{tr}\left[Q \mathscr{R}_{\nu}(y) \mathscr{R}_{\lambda}(z)\right]\right\} .
\end{aligned}
$$

We see that, as a consequence of eq. (3.14), when evaluating the triangle diagrams we do not need to insert the whole current $j_{\mu}(x)$ (3.5) (in the vertex which is acted upon by the derivative) but only the lowest dimensional part $j_{3}^{\mu}(x)[26]$. This is not yet sufficient to prove the independence of $L(x) W$ on the parameters $c_{k}$ of $\mathcal{L}_{6}$, since they still appear in the combinations $\mathscr{L}_{\mu}$ and $\mathscr{R}_{\mu}$ at the other two vertices of the triangle.

To evaluate the correlators $\partial^{\mu} W_{\mu \nu \lambda}^{X Y Z}(x, y, z)$ of eq. (3.15) we first regularize the effective action $W$ by using dimensional regularization [34-36] with the t'Hooft-Veltman prescription for $\gamma_{5}{ }^{7}$ In momentum space the correlators are easily computed through well-known triangle diagrams. Denoting by $W_{\mathrm{r}}$ the regulated effective action, we get:

$$
\begin{aligned}
L(x) W_{\mathrm{r}}=-\frac{1}{24 \pi^{2}} \varepsilon^{\mu \nu \rho \sigma} & \left\{\xi_{A A} \partial_{\mu} A_{\nu}(x) \cdot \partial_{\rho} A_{\sigma}(x)\right. \\
& +i \xi_{\varphi A} \partial_{\mu}\left(\varphi^{\dagger} \stackrel{\leftrightarrow}{D}_{\nu} \varphi\right)(x) \cdot \partial_{\rho} A_{\sigma}(x) \\
& \left.-\xi_{\varphi \varphi} \partial_{\mu}\left(\varphi^{\dagger} \stackrel{\leftrightarrow}{D}_{\nu} \varphi\right)(x) \cdot \partial_{\rho}\left(\varphi^{\dagger} \stackrel{\leftrightarrow}{D}_{\sigma} \varphi\right)(x)\right\}
\end{aligned}
$$

where:

$$
\begin{aligned}
& \xi_{A A}=g^{2} \operatorname{tr} Q^{3} \\
& \xi_{\varphi A}=2 g \operatorname{tr} Q^{2}\left(C_{\phi R}-C_{\phi L}\right) \\
& \xi_{\varphi \varphi}=\operatorname{tr} Q\left(C_{\phi R}-C_{\phi L}\right)^{2} .
\end{aligned}
$$

\footnotetext{
${ }^{7}$ That is: $\left\{\gamma_{5}, \gamma^{\bar{\mu}}\right\}=0$ and $\left[\gamma_{5}, \gamma^{\hat{\mu}}\right]=0, \gamma^{\bar{\mu}}\left(\gamma^{\hat{\mu}}\right)$ denoting the four-dimensional $((d-4)$-dimensional $)$ part of $\gamma^{\mu}$. Note that such prescription is at the origin of chirality-mixing contribution: terms like $(1-$ $\left.\gamma_{5}\right) k \gamma^{\hat{\mu}}\left(1+\gamma_{5}\right)$ do not vanish and give rise to evanescent terms of order $(d-4)$. Due to the pole $1 /(d-4)$ arising from the integration, such terms are converted into finite, chirally-mixed terms.
} 
As a consequence, the gauge variation of $W_{\mathrm{r}}$ is given by:

$$
\delta_{\alpha} W_{\mathrm{r}}=\int d^{4} x \alpha(x) L(x) W_{\mathrm{r}},
$$

and we see that it is still dependent on the coefficients $c_{k}$ through the combinations $\xi_{\varphi A}$ and $\xi_{\varphi \varphi}$. However, the right-hand side of this expression should not be identified with the anomaly, since the effective action $W$ and the functional $W_{\mathrm{r}}$ differ by counterterms, space-time integrals of local polynomials in the bosonic fields, that can be added to $W_{\mathrm{r}}$. If a counterterm $W_{\mathrm{c}}$ exists such that $\delta_{\alpha} W_{\mathrm{c}}+\delta_{\alpha} W_{\mathrm{r}}=0$, we can define $W=W_{\mathrm{r}}+W_{\mathrm{c}}$ and the theory is free from gauge anomalies. In general, we expect $L(x) W_{\mathrm{r}}$ to contain relevant and irrelevant contributions. This is the case in the model under consideration. The first line in eq. (3.16) is relevant. A local counterterm whose gauge variation cancels this term does not exist. Gauge anomaly cancellation requires the condition $\operatorname{tr} Q^{3}=0$, satisfied by construction in this model. The remaining part of $L(x) W_{\mathrm{r}}$ is irrelevant. Indeed consider:

$$
\begin{aligned}
W_{\mathrm{c}}=\frac{1}{24 \pi^{2}} \varepsilon^{\mu \nu \rho \sigma} \int d^{4} y\{ & +i \xi_{\varphi A} g A_{\mu}(y)\left(\varphi^{\dagger} \stackrel{\leftrightarrow}{D}_{\nu} \varphi\right)(y) \cdot \partial_{\rho} A_{\sigma}(y) \\
& \left.-\xi_{\varphi \varphi} g A_{\mu}(y)\left(\varphi^{\dagger} \stackrel{\leftrightarrow}{D}_{\nu} \varphi\right)(y) \cdot \partial_{\rho}\left(\varphi^{\dagger} \stackrel{\leftrightarrow}{D}_{\sigma} \varphi\right)(y)\right\} .
\end{aligned}
$$

We have:

$$
\begin{aligned}
L(x) W_{\mathrm{c}}=-\frac{1}{24 \pi^{2}} \varepsilon^{\mu \nu \rho \sigma} \int d^{4} y\{ & +i \xi_{\varphi A} \partial_{\mu} \delta^{4}(x-y)\left(\varphi^{\dagger} \overleftrightarrow{D}_{\nu} \varphi\right)(y) \cdot \partial_{\rho} A_{\sigma}(y) \\
& \left.-\xi_{\varphi \varphi} \partial_{\mu} \delta^{4}(x-y)\left(\varphi^{\dagger} \overleftrightarrow{D}_{\nu} \varphi\right)(y) \cdot \partial_{\rho}\left(\varphi^{\dagger} \stackrel{\leftrightarrow}{D}_{\sigma} \varphi\right)(y)\right\} \\
=\frac{1}{24 \pi^{2}} \varepsilon^{\mu \nu \rho \sigma}\{ & +i \xi_{\varphi A} \partial_{\mu}\left(\varphi^{\dagger} \stackrel{\leftrightarrow}{D}_{\nu} \varphi\right)(x) \cdot \partial_{\rho} A_{\sigma}(x) \\
& \left.-\xi_{\varphi \varphi} \partial_{\mu}\left(\varphi^{\dagger} \stackrel{\leftrightarrow}{D}_{\nu} \varphi\right)(x) \cdot \partial_{\rho}\left(\varphi^{\dagger} \stackrel{\leftrightarrow}{D}_{\sigma} \varphi\right)(x)\right\} .
\end{aligned}
$$

We see that $L(x) W_{\mathrm{c}}$ cancels the second and third lines in eq. (3.16). Hence, by choosing $W=W_{\mathrm{r}}+W_{\mathrm{c}}$ we end up with:

$$
L(x) W=-\frac{g^{2} \operatorname{tr} Q^{3}}{24 \pi^{2}} \varepsilon^{\mu \nu \rho \sigma} \partial_{\mu} A_{\nu}(x) \cdot \partial_{\rho} A_{\sigma}(x) .
$$

Thus the condition for gauge anomaly cancellation has no dependence on the coefficients of the higher dimensional operators defining the EFT. It is completely controlled by the charge assignment $Q$ of the fermion sector. Some comments are in order.

- The gauge invariance of the combination $i\left(\varphi^{\dagger} D_{\nu} \varphi-D_{\mu} \varphi^{\dagger} \varphi\right)$ is a crucial ingredient for the result (3.20).

- Non-trivial gauge-invariant local polynomials like those of eq. (2.9), which would have contributed to the anomaly, have not shown up in $L(x) W_{\mathrm{r}}$.

- The result (3.20) holds whether the gauge symmetry is realized in the unbroken phase or not. If the theory is in the broken phase, the whole combination $i\left(\varphi^{\dagger} D_{\nu} \varphi-D_{\mu} \varphi^{\dagger} \varphi\right)$ should be included in $L(x) W_{\mathrm{r}}$. Had we kept only the first term of this expression in 
the expansion around the vacuum $\varphi=v / \sqrt{2}$, that is $i\left(\varphi^{\dagger} D_{\nu} \varphi-D_{\mu} \varphi^{\dagger} \varphi\right)=v^{2} g A_{\mu}+\ldots$, the second and third lines in eq. (3.16) would have collapsed to expressions proportional to the first line and we would have missed the cancellation displayed above.

- The $c_{k}$-dependent terms shown in eqs. (3.16) and (3.19) depend on the regularization used. For example, if we compute $L(x) W_{\mathrm{r}}$ using a set of Pauli-Villars regulators [37], we get different coefficients $\xi_{\varphi A}$ and $\xi_{\varphi \varphi}$. In this case, the same cancellation mechanism is at work, but with a different choice of counterterms. This reflects the unphysical nature of the $c_{k}$-dependent part. Only the sum of the regulated diagrams and counterterms is a physical quantity. This has a direct impact on the amplitude for a physical process, evaluated beyond the tree-level approximation. If we do not include the contribution of the counterterm $W_{\mathrm{c}}$ to the set of relevant diagrams, in general we will get a gauge-dependent result. Hence the counterterms highlighted above have not a purely academic interest, but play an essential role in the evaluation of a physical quantity.

To complete the discussion, we still need to include the contribution from four-fermion operators, which we do in the next section.

\subsection{Four-fermion operators}

Four-fermion operators can be easily accounted for, without spoiling our conclusion. Consider a complete set of four-fermion operators. Here again we focus on the $B$ and $L$ conserving sector. Through Fierz transformations we can cast their contribution to the Lagrangian in the form:

$$
\mathcal{L}_{6}^{4 F}=\frac{1}{2} \bar{l}_{I} l C_{I J}^{l l} \bar{l} \Gamma_{J} l+\frac{1}{2} \bar{q} \Gamma_{I} q C_{I J}^{q q} \bar{q} \Gamma_{J} q+\bar{l} \Gamma_{I} l C_{I J}^{l q} \bar{q} \Gamma_{J} q,
$$

where $\Gamma_{I}$ represents the set $\left(1, \gamma_{5}, \gamma^{\mu}, \gamma^{\mu} \gamma_{5}, \sigma^{\mu \nu}\right)$ and $C_{I J}^{l l, q q, q l}$ are matrices of coefficients. We can equivalently express the combination in (3.21) in terms of fermion bilinears, by making use of a set of bosonic auxiliary fields $\chi_{I}=\left(s, p, v^{\mu}, a^{\mu}, t^{\mu \nu}\right)$ with suitable masses and couplings [24]:

$$
\mathcal{L}_{4}^{\mathrm{aux}}=\chi_{I} \bar{f}\left(\begin{array}{cc}
X_{I J}^{l} & 0 \\
0 & X_{I J}^{q}
\end{array}\right) \Gamma_{J} f-\frac{1}{2} \chi_{I} M_{I}^{2} \chi_{I} .
$$

We can always choose $X_{I J}^{l, q}$ and $M_{I}^{2}$ such that, by eliminating the auxiliary fields $\chi_{I}$ through their equations of motion, we reproduce eq. (3.21). Notice that the gauge invariance of $\mathcal{L}_{6}^{4 F}$ implies the gauge invariance of all the auxiliary fields $\chi_{I}$ in $\mathcal{L}_{4}^{\text {aux }}$. The new term $\mathcal{L}_{4}^{\text {aux }}$ modifies the bilinear fermion interaction of eq. (3.10) by adding to the combinations $\mathscr{S}$, $\mathscr{P}, \mathscr{V}_{\mu}, \mathscr{A}_{\mu}$ and $\mathscr{T}_{\mu \nu}$ new gauge invariant contributions. In particular, the quantities $\mathscr{V}_{\mu}$ and $\mathscr{A}_{\mu}$, relevant to the computation of $L(x) W$ are now modified into:

$$
\begin{aligned}
& \mathscr{V}_{\mu}=\frac{1}{2}\left[-g Q A_{\mu}+i\left(C_{\phi R}+C_{\phi L}\right)\left(\varphi^{\dagger} \stackrel{\leftrightarrow}{D}_{\mu} \varphi\right)+C_{V} v_{\mu}\right] \\
& \mathscr{A}_{\mu}=\frac{1}{2}\left[+g Q A_{\mu}+i\left(C_{\phi R}-C_{\phi L}\right)\left(\varphi^{\dagger} \stackrel{\leftrightarrow}{D}_{\mu} \varphi\right)+C_{A} a_{\mu}\right],
\end{aligned}
$$


where $C_{V, A}$ are matrices easily identifiable from the couplings $X_{I J}^{l, q}$. The effective action $W$ now depends on the bosonic background through the gauge field $A_{\mu}$ and the gauge invariant quantities $i\left(\varphi^{\dagger} D_{\nu} \varphi-D_{\mu} \varphi^{\dagger} \varphi\right), v_{\mu}$ and $a_{\mu}$. The gauge variation $L(x) W_{\mathrm{r}}$ is a linear combination of:

$$
\varepsilon^{\mu \nu \rho \sigma} \partial_{\mu} B_{\nu}^{a}(x) \cdot \partial_{\rho} B_{\sigma}^{b}(x),
$$

where $B_{\mu}^{a}=\left(A_{\mu}, i\left(\varphi^{\dagger} D_{\nu} \varphi-D_{\mu} \varphi^{\dagger} \varphi\right), v_{\mu}, a_{\mu}\right)$. Only the term with $B_{\mu}^{a}=B_{\mu}^{b}=A_{\mu}$ is nontrivial. By following the same steps of the previous section, we see that the rest is irrelevant and we conclude that the presence of four-fermion interactions does not alter the condition of gauge anomaly cancellation.

\section{Discussion}

There are several arguments supporting the idea that the cancellation of gauge anomalies in a general EFT only depends on the set of fermion representations and not on the features of the non-renormalizable sector. Nevertheless, an explicit computation of the gauge anomaly in a non-trivial EFT, including the full set of allowed operators up to a given dimensionality and allowing for the most general bosonic background is still missing. This note was meant to fill this gap and to elucidate the mechanism that removes the dependence of the anomaly on the higher dimensional operators. An additional motivation is provided by the ineffectiveness of cohomological arguments to rule out contributions to the anomaly of arbitrarily high dimension. Indeed, when the gauge group is non semi-simple, candidate anomalies are local gauge-invariant polynomials whose dimension is in principle unbounded.

In this note we have carried out an explicit computation of the anomaly in a simple abelian model, with several features in common with the SMEFT. We have included the most general set of dimension six operators comprising also four-fermion operators. We have evaluated the anomaly within dimensional regularization both for diagrams involving minimal fermion interactions and for those where fermions are non-minimally coupled. After the inclusion of appropriate counterterms, the resulting anomaly is independent on the coefficients of the non-renormalizable sector. Counterterms are expected to depend on the adopted regularization and only the sum of all diagrams, counterterms included, has a physical meaning. Thus the counterterms highlighted here have a direct impact when amplitudes for physical processes are evaluated beyond the tree-level approximation within dimensional regularization.

We can identify two crucial ingredients in our derivation. Firstly, in the presence of the higher-dimensional operators, the gauge current acquires a new gauge invariant term, that does not contribute to the gauge variation of the effective action. This point has been recently stressed in ref. [26]. Secondly, the variation of the effective action evaluated in a general bosonic background includes relevant and irrelevant terms. Only the irrelevant component depends on the coefficients of the non-renormalizable operators. This component can be subtracted by adding a local counterterm to the Lagrangian density. We expect that also in the SMEFT a similar mechanism takes place. Indeed we do not foresee a qualitatively different behaviour of the SMEFT under a generic gauge transformation. Nevertheless, we think that it is important to fully identify the set of counterterms needed 
to cancel the spurious non-invariance induced by higher-dimensional SMEFT operators. Indeed, counterterms of this type have already been adopted in the automation of one-loop computations in the SMEFT, on a case by case basis [38, 39]. The general computation illustrated in this note might allow to determine the whole set of such counterterms, covering all possible processes involving operators of dimension six.

\section{Acknowledgments}

I thank Cen Zhang for signaling to me the works of refs. [38, 39]. This project has received support by INFN and by the European Union's Horizon 2020 research and innovation programme under the Marie Sklodowska-Curie grant agreement $N^{\circ}$ 860881-HIDDeN.

Open Access. This article is distributed under the terms of the Creative Commons Attribution License (CC-BY 4.0), which permits any use, distribution and reproduction in any medium, provided the original author(s) and source are credited.

\section{References}

[1] A. Adams, N. Arkani-Hamed, S. Dubovsky, A. Nicolis and R. Rattazzi, Causality, analyticity and an IR obstruction to UV completion, JHEP 10 (2006) 014 [hep-th/0602178] [INSPIRE].

[2] L. Vecchi, Causal versus analytic constraints on anomalous quartic gauge couplings, JHEP 11 (2007) 054 [arXiv:0704.1900] [INSPIRE].

[3] A. Nicolis, R. Rattazzi and E. Trincherini, Energy's and amplitudes' positivity, JHEP 05 (2010) 095 [Erratum ibid. 11 (2011) 128] [arXiv:0912.4258] [INSPIRE].

[4] B. Bellazzini, Softness and amplitudes' positivity for spinning particles, JHEP 02 (2017) 034 [arXiv: 1605.06111] [INSPIRE].

[5] C. de Rham, S. Melville, A.J. Tolley and S.-Y. Zhou, Positivity bounds for scalar field theories, Phys. Rev. D 96 (2017) 081702 [arXiv:1702.06134] [InSPIRE].

[6] C. de Rham, S. Melville, A.J. Tolley and S.-Y. Zhou, UV complete me: positivity bounds for particles with spin, JHEP 03 (2018) 011 [arXiv:1706.02712] [INSPIRE].

[7] C. Zhang and S.-Y. Zhou, Positivity bounds on vector boson scattering at the LHC, Phys. Rev. D 100 (2019) 095003 [arXiv: 1808.00010] [INSPIRE].

[8] Q. Bi, C. Zhang and S.-Y. Zhou, Positivity constraints on aQGC: carving out the physical parameter space, JHEP 06 (2019) 137 [arXiv: 1902.08977] [INSPIRE].

[9] B. Bellazzini, J. Elias Miró, R. Rattazzi, M. Riembau and F. Riva, Positive moments for scattering amplitudes, arXiv:2011.00037 [INSPIRE].

[10] G.N. Remmen and N.L. Rodd, Consistency of the Standard Model effective field theory, JHEP 12 (2019) 032 [arXiv:1908.09845] [INSPIRE].

[11] G.N. Remmen and N.L. Rodd, Signs, spin, SMEFT: positivity at dimension six, arXiv:2010.04723 [INSPIRE].

[12] C. Zhang and S.-Y. Zhou, Convex geometry perspective on the (Standard Model) effective field theory space, Phys. Rev. Lett. 125 (2020) 201601 [arXiv:2005.03047] [INSPIRE]. 
[13] S.L. Adler, Axial vector vertex in spinor electrodynamics, Phys. Rev. 177 (1969) 2426 [INSPIRE].

[14] J.S. Bell and R. Jackiw, A PCAC puzzle: $\pi^{0} \rightarrow \gamma \gamma$ in the $\sigma$ model, Nuovo Cim. A 60 (1969) 47 [INSPIRE].

[15] H. Georgi and S.L. Glashow, Gauge theories without anomalies, Phys. Rev. D 6 (1972) 429 [INSPIRE].

[16] G. Barnich and M. Henneaux, Renormalization of gauge invariant operators and anomalies in Yang-Mills theory, Phys. Rev. Lett. 72 (1994) 1588 [hep-th/9312206] [INSPIRE].

[17] G. Barnich, F. Brandt and M. Henneaux, Local BRST cohomology in gauge theories, Phys. Rept. 338 (2000) 439 [hep-th/0002245] [INSPIRE].

[18] J.A. Dixon and M. Ramon Medrano, Anomalies in the operator product expansion, Phys. Rev. D 22 (1980) 429 [inSPIRE].

[19] J.A. Dixon, Anomalies, BRS cohomology and effective theories, Phys. Rev. Lett. 67 (1991) 797 [INSPIRE].

[20] S. Marculescu and L. Mezincescu, Axial anomaly in nonrenormalizable theories, preprint IFIN-FT-162-1978, Bucarest, Romania (1978).

[21] Y. Kim, P.Y. Pac and H.K. Shin, Spinor loop anomalies in higher derivative theories, Phys. Rev. D 39 (1989) 1251 [INSPIRE].

[22] J. Minn, J. Kim and C.-K. Lee, Spinor loop anomalies with very general local fermion Lagrangians, Phys. Rev. D 35 (1987) 1872 [INSPIRE].

[23] L. Álvarez-Gaumé and E. Witten, Gravitational anomalies, Nucl. Phys. B 234 (1984) 269 [INSPIRE].

[24] J. Soto, Anomaly cancellation at finite cutoff, Phys. Rev. D 45 (1992) 4621 [InSPIRE].

[25] O. Catà, W. Kilian and N. Kreher, Gauge anomalies in the Standard-Model effective field theory, arXiv:2011.09976 [INSPIRE].

[26] Q. Bonnefoy, L. Di Luzio, C. Grojean, A. Paul and A.N. Rossia, Comments on gauge anomalies at dimension-six in the Standard Model effective field theory, arXiv:2012.07740 [INSPIRE].

[27] S.L. Adler and W.A. Bardeen, Absence of higher order corrections in the anomalous axial vector divergence equation, Phys. Rev. 182 (1969) 1517 [INSPIRE].

[28] D. Anselmi, Adler-Bardeen theorem and cancellation of gauge anomalies to all orders in nonrenormalizable theories, Phys. Rev. D 91 (2015) 105016 [arXiv:1501.07014] [INSPIRE].

[29] J. Wess and B. Zumino, Consequences of anomalous Ward identities, Phys. Lett. B 37 (1971) 95 [INSPIRE].

[30] B. Grzadkowski, M. Iskrzynski, M. Misiak and J. Rosiek, Dimension-six terms in the Standard Model Lagrangian, JHEP 10 (2010) 085 [arXiv: 1008.4884] [INSPIRE].

[31] W.A. Bardeen, Anomalous Ward identities in spinor field theories, Phys. Rev. 184 (1969) 1848 [INSPIRE].

[32] T.E. Clark and S.T. Love, The axial anomaly and antisymmetric tensor fields, Nucl. Phys. B 223 (1983) 135 [INSPIRE]. 
[33] W.A. Bardeen and N. Deo, Comment on spinor anomalies, Nucl. Phys. B 264 (1986) 364 [INSPIRE].

[34] G. 't Hooft and M.J.G. Veltman, Regularization and renormalization of gauge fields, Nucl. Phys. B 44 (1972) 189 [InSPIRE].

[35] T. Marinucci and M. Tonin, Dimensional regularization and anomalies, Nuovo Cim. A 31 (1976) 381 [INSPIRE].

[36] P. Breitenlohner and D. Maison, Dimensional renormalization and the action principle, Commun. Math. Phys. 52 (1977) 11 [inSPIRE].

[37] A. Bilal, Lectures on anomalies, arXiv:0802.0634 [INSPIRE].

[38] G. Durieux, J. Gu, E. Vryonidou and C. Zhang, Probing top-quark couplings indirectly at Higgs factories, Chin. Phys. C 42 (2018) 123107 [arXiv: 1809.03520] [InSPIRE].

[39] C. Degrande, G. Durieux, F. Maltoni, K. Mimasu, E. Vryonidou and C. Zhang, Automated one-loop computations in the SMEFT, arXiv:2008.11743 [INSPIRE]. 